\title{
Adaptive visible and short-wave infrared camouflage using a dynamically tunable metasurface
}

\author{
Ebru Buhara, ${ }^{1,2, *}$ Amir Ghobadi, ${ }^{1,2}$ (1) and Ekmel Ozbay ${ }^{1,2,3,4}$ \\ ${ }^{1}$ Department of Electrical and Electronics Engineering, Bilkent University, 06800 Ankara, Turkey \\ ${ }^{2}$ NANOTAM-Nanotechnology Research Center, Bilkent University, 06800 Ankara, Turkey \\ ${ }^{3}$ UNAM-National Nanotechnology Research Center, Institute of Materials Science and Nanotechnology, Bilkent University, 06800 Ankara, Turkey \\ ${ }^{4}$ Department of Physics, Bilkent University, 06800 Ankara, Turkey \\ *Corresponding author: ebru.buhara@bilkent.edu.tr
}

Received 2 August 2021; revised 26 August 2021; accepted 27 August 2021; posted 27 August 2021 (Doc. ID 439435); published 23 September 2021

In this Letter, a dynamically tunable metasurface, which is based on antimony trisulfide, is introduced. In this structure, first a metal-insulator-metal (MIM) nanocavity is optimized in a way that, upon phase change, the visible response switches from a transmissive colored window into a reflective mirror. Later, an indium tin oxide nanoantenna is integrated on the MIM cavity to provide antireflection in the short-wave infrared (SWIR) range for SWIR mode thermal camouflage. The MIM host acts as a thermally tunable substrate to tune the SWIR response of the design. () 2021 Optical Society of America

https://doi.org/10.1364/OL.439435

Active metasurfaces with a dynamically tunable optical response have been a long-standing goal because they promise extendable and reconfigurable functionalities [1]. Among the different applications, tunable color filters are some of the most promising applications of active metasurfaces [2]. A commonly used approach to fabricate actively tunable color filters is to use phase change materials (PCMs), such as vanadium dioxide [3,4], germanium-antimony-tellurium [5], germanium telluride [6], and antimony trisulfide $\left(\mathrm{Sb}_{2} \mathrm{~S}_{3}\right)$ [7]. Among these, $\mathrm{Sb}_{2} \mathrm{~S}_{3}$ with an amorphous phase in the lower temperatures and a crystalline phase in the higher temperatures $\left(>270^{\circ}\right)$ [8], is an ideal material for a thermally tunable color generation, due to its large index contrast between two phases in the visible range.

The equivalence of the color adoption in the longer ranges, such as the infrared region, is thermal camouflage [9]. Therefore, similarly, these PCM-based metasurfaces are used for adaptive thermal camouflage [10-12]. In general, three thermal camera types are used to detect an object. Short-wave infrared (SWIR, 900-1700 nm), mid-wave infrared (MWIR, 3-5 $\mu \mathrm{m}$ ), and long-wave infrared (LWIR, 8-12 $\mu \mathrm{m}$ ) modes. MWIR and LWIR mode cameras detect the blackbody radiation from a hot matter, while the SWIR mode detects the reflected solar irradiation from an object. The manipulation of the absorption spectrum of a nanoantenna is used as a means to design a proper thermal emitter $[13,14]$. The proposed metasurfaces are designed in a way that, in the hot state, the thermal signatures in the MWIR and LWIR ranges are mitigated. This is achieved by the transition from the dielectric to the metal phase with a dominant reflectance response in the above-mentioned ranges or placing emission resonance in the non-transmissive window $(5-8 \mu \mathrm{m})$ in the hot state $[10,15]$. However, to the best of our knowledge, there is no work on the use of PCMs for SWIR mode camouflage where, unlike the MWIR and LWIR, the solar reflection should be reduced.

In this Letter, a metasurface design is developed to provide adaptive camouflage in both visible and SWIR ranges. The proposed metasurface is made of an indium tin oxide (ITO) grating on a metal-insulator-metal (MIM, $\mathrm{Ag}-\mathrm{Sb}_{2} \mathrm{~S}_{3}-\mathrm{Ag}$ ) nanocavity. In the amorphous state, the design operates as a colored transmissive window while, in the crystalline phase, it switches into a reflective mirror. In the meantime, the cavity acts as a thermally tunable host for the ITO nanoantenna providing tunable SWIR absorption to cover two transmissive regions at $1150-1350 \mathrm{~nm}$ (Region I) and 1400-1700 nm (Region II). It is found that the excitation of extended surface plasmons (ESPs) and guided mode resonances (GMRs) are responsible for light absorption in the SWIR range. Our theoretical calculations show that, besides the design's ability for color adoption, the SWIR reflectance in Region I/Region II are reduced to $0.37 / 0.53$ and $0.75 / 0.25$ in the amorphous/crystalline phases.

To begin with, first the MIM cavity is optimized to achieve a mirror/window transition upon phase transition. The top and bottom metals are Ag (Johnson and Christy model [16]) with an identical thickness of $\mathrm{D}_{M}$. The insulator is $\mathrm{Sb}_{2} \mathrm{~S}_{3}$ with a thickness of $\mathrm{D}_{I}$ and the permittivity data obtained from a previous study [8]. Simulations are performed using the Lumerical Finite-Difference Time-Domain software [17] For this aim, we first fix the $\mathrm{D}_{I}$ in $40 \mathrm{~nm}$, and sweep the $\mathrm{D}_{M}$ to find the best metal thickness. As shown in Figs. 1(a) and 1(b), as the metal becomes thicker, the full-width at half-maximum (FWHM) of the transmission resonance gets narrower. However, for layers thicker than $15 \mathrm{~nm}$, the transmission amplitude also reduces. Therefore, in a $\mathrm{D}_{M}=15 \mathrm{~nm}$, we have a right trade-off between 
(a)

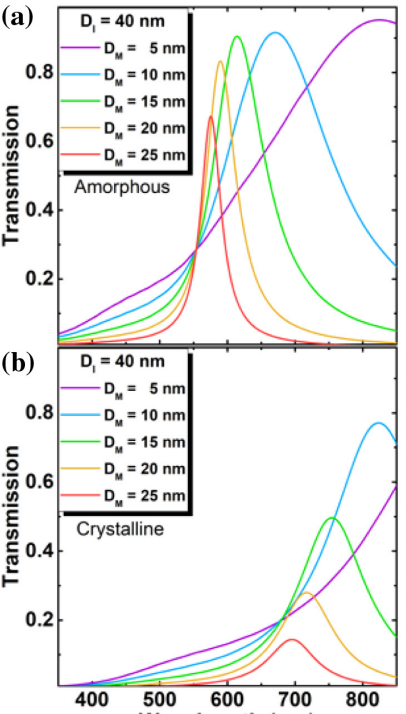

(e)
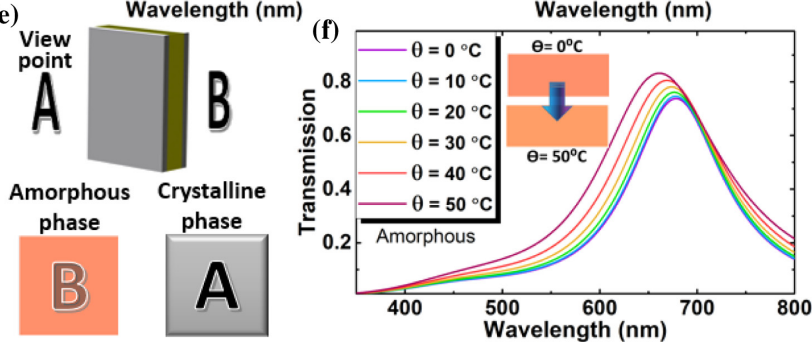

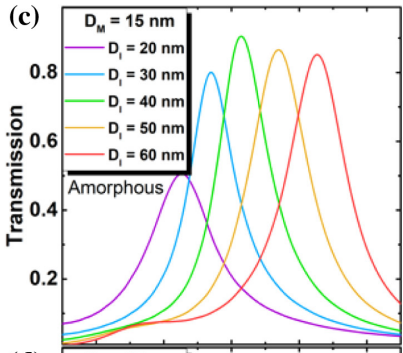

(d)

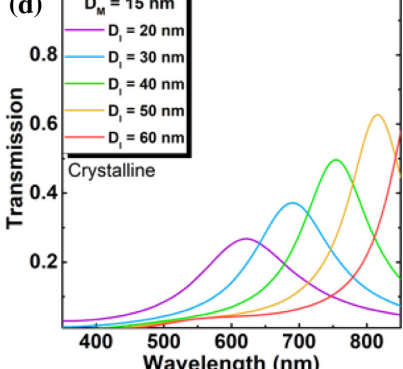

Wavelength $(\mathrm{nm})$

Fig. 1. Transmission spectra of the MIM cavity for different $\mathrm{D}_{M}$ values for the (a) amorphous and (b) crystalline phases. The impact of spacer thickness $\left(\mathrm{D}_{I}\right)$ on the transmission spectra of the MIM cavity design for the (c) amorphous and (d) crystalline phases. (e) Schematic representation of the optical response of the MIM cavity in the visible range upon phase transition from amorphous to crystalline. (f) Angular response of the MIM cavity.

the amplitude and FWHM of the transmissive color filter. Next, we fixed the metal layer thickness in its optimal value of $15 \mathrm{~nm}$ and tune the spacer thickness to find the proper dimension to have mirror-window transition, as shown in Figs. 1(c) and 1 (d). For this, the $\mathrm{D}_{I}$ thickness is swept from 20 to $60 \mathrm{~nm}$. The resonance wavelength of the amorphous phase MIM cavity is located at $670 \mathrm{~nm}$, while the design is dominantly reflective in the crystalline mode. Therefore, in these optimized geometries, the MIM cavity acts as a mirror in the hot phase and a colored window in the cold state. This has been schematically shown in Fig. 1(e). Moreover, the proposed design retains its color over wide incident angles. As depicted in Fig. 1(f),

In the next step, this functionality should be extended toward the SWIR range to provide antireflection in the $900-1700 \mathrm{~nm}$ range. For this, the MIM cavity is coupled into a grating design to excite the resonant modes in the SWIR. In the meantime, the grating material should not disturb the visible response of the MIM cavity. This requirement can be satisfied with the ITO material. Besides building a resonant absorptive metasurface in the SWIR range, to further suppress the reflection in this range, higher-order Fabry-Perot (FP) modes can also be excited. Therefore, the cavity is redesigned to have resonant transmission in both visible and SWIR ranges. For this aim, the spacer layer thickness is increased to $155 \mathrm{~nm}$. The schematic representation of the design is presented in Fig. 2(a). The ITO grating layer

has a width of $\mathrm{W}$, height of $\mathrm{H}$, and periodicity of $\mathrm{P}$. These three dimensions are optimized to achieve resonant absorptions at Region I (for amorphous phase) and Region II (for crystalline phase). The optimized design has a periodicity of $1060 \mathrm{~nm}$, grating width of $420 \mathrm{~nm}$, and height of $250 \mathrm{~nm}$. The spectral reflection (R) and transmission (T) data of the metasurface in both phases are depicted in Fig. 2(b). In the amorphous phase, the SWIR response consists of three resonant dips located at $\lambda_{1}=1131 \mathrm{~nm}, \lambda_{2}=1275 \mathrm{~nm}$, and $\lambda_{3}=1338 \mathrm{~nm}$. Upon the phase change, the resonances are redshifted to $\lambda_{1}^{\prime}=1156 \mathrm{~nm}$, $\lambda_{2}^{\prime}=1550 \mathrm{~nm}$, and $\lambda_{3}^{\prime}=1579 \mathrm{~nm}$. In the meantime, the visible light transmission response is quite similar to the MIM cavity design, which is expected considering the transparency of ITO and its small filling fraction. Considering the scenario shown in Fig. 2(c), the proposed metasurface can simultaneously satisfy the visible and SWIR mode camouflage requirements. In the hot state, the metasurface is a purple-like mirror with antireflection capability in Region II of the SWIR range. In the cold state, the design turns into an orange transmissive color filter, and the SWIR response is moved to Region I, as shown in Fig. 2(d). The angular response of the design has also been studied to examine the tolerance of the resonance modes against incidence angle change. As Fig. 2(e) implies, the visible response that defines the color of the metasurface is almost intact in oblique irradiation. However, the first and third SWIR resonance modes are greatly affected with the angle of incidence. The first mode blueshifts, while the third mode experiences a redshift.

To scrutinize the impact of geometries, the parametric sweeps are performed on the $\mathrm{W}, \mathrm{H}$, and $\mathrm{P}$ of the grating, only for the amorphous phase design. Figure 2(f) shows the reflection contour plot as a function of P. From this panel, the second resonant $\operatorname{dip}\left(\lambda_{2}\right)$ is insensitive to the periodicity. However, other modes, i.e., $\lambda_{1}$, and $\lambda_{3}$, slightly shift to longer wavelengths, as the $\mathrm{P}$ widens. Moreover, their resonance strength is also reduced. Therefore, the $\mathrm{P}$ value is fixed at $1060 \mathrm{~nm}$ to have all three resonances spectrally adjacent and to provide a broadband antireflection to cover the Region I. The other parameter is $\mathrm{H}$. As plotted in Fig. 2(g), the reflection dips spectral position has slight sensitivity to the grating height. However, as the grating thickens, new resonant ripples start to appear in the visible range, and this diminishes the color purity of the design. Moreover, in the longer ranges $(1600-1800 \mathrm{~nm})$, a thicker grating causes less reflection, which is a desired response for SWIR mode camouflage. Therefore, the grating height is chosen as $250 \mathrm{~nm}$ to have a fair trade-off between color purity and long-range SWIR antireflection. Finally, the impact of the W on different resonance modes is studied. As shown in Fig. 2(h), this parameter dominantly affects the first resonance. The wider grating shifts the first mode toward longer wavelengths but, in the meantime, it reduces its resonance strength. Moreover, in the visible range, unwanted resonances start to appear and get boarded as we go toward larger Ws. To gain an insight into the origin of these resonance modes, the electric field (E-field) and absorption profiles are extracted across the design cross section. Figures 3(a) and 3(b) show the E-field and absorption profiles across the design in the $\lambda_{1}=1131 \mathrm{~nm}$. Based on $[18,19]$, this field profile shows the simultaneous excitation of ESPs in the metal-insulator interface and GMRs excited inside the cavity design. The ITO grating acts as a cladding layer to excite the GMRs inside the MIM core Therefore, the change in grating 

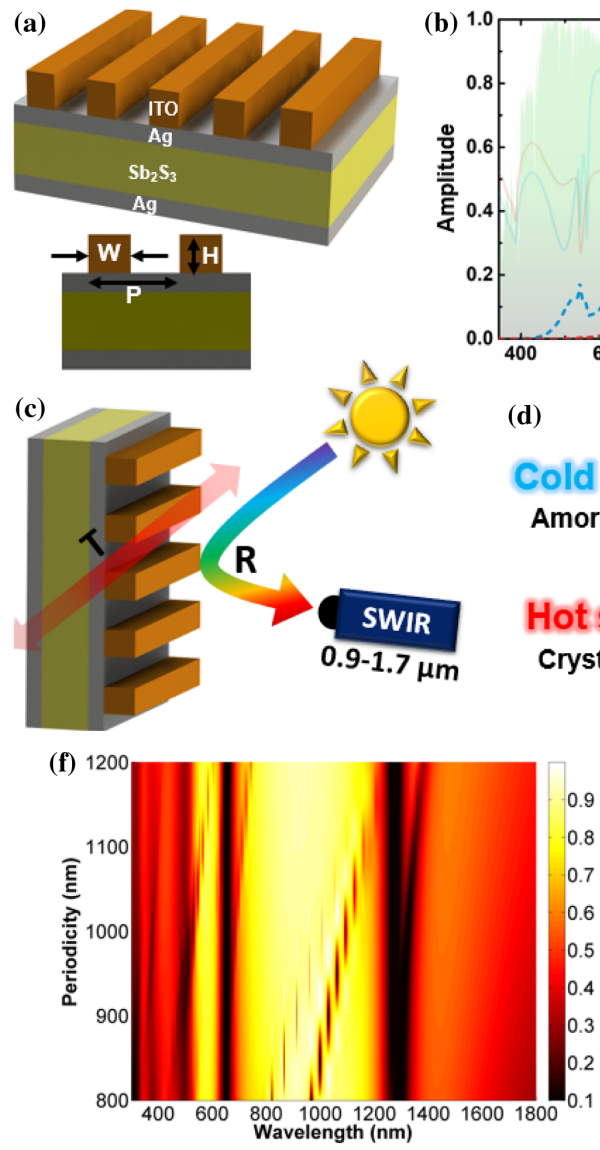

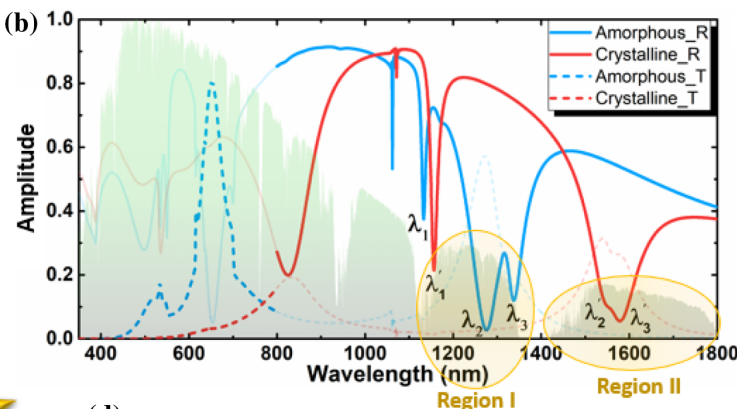

(d)
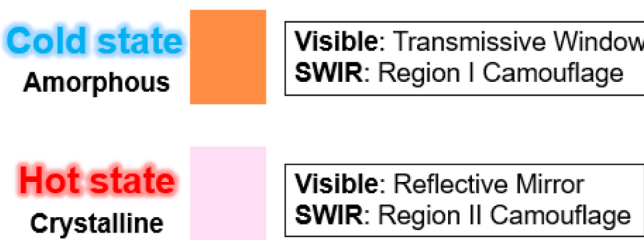

Visible: Transmissive Window SWIR: Region I Camouflage SWIR: Region II Camouflage
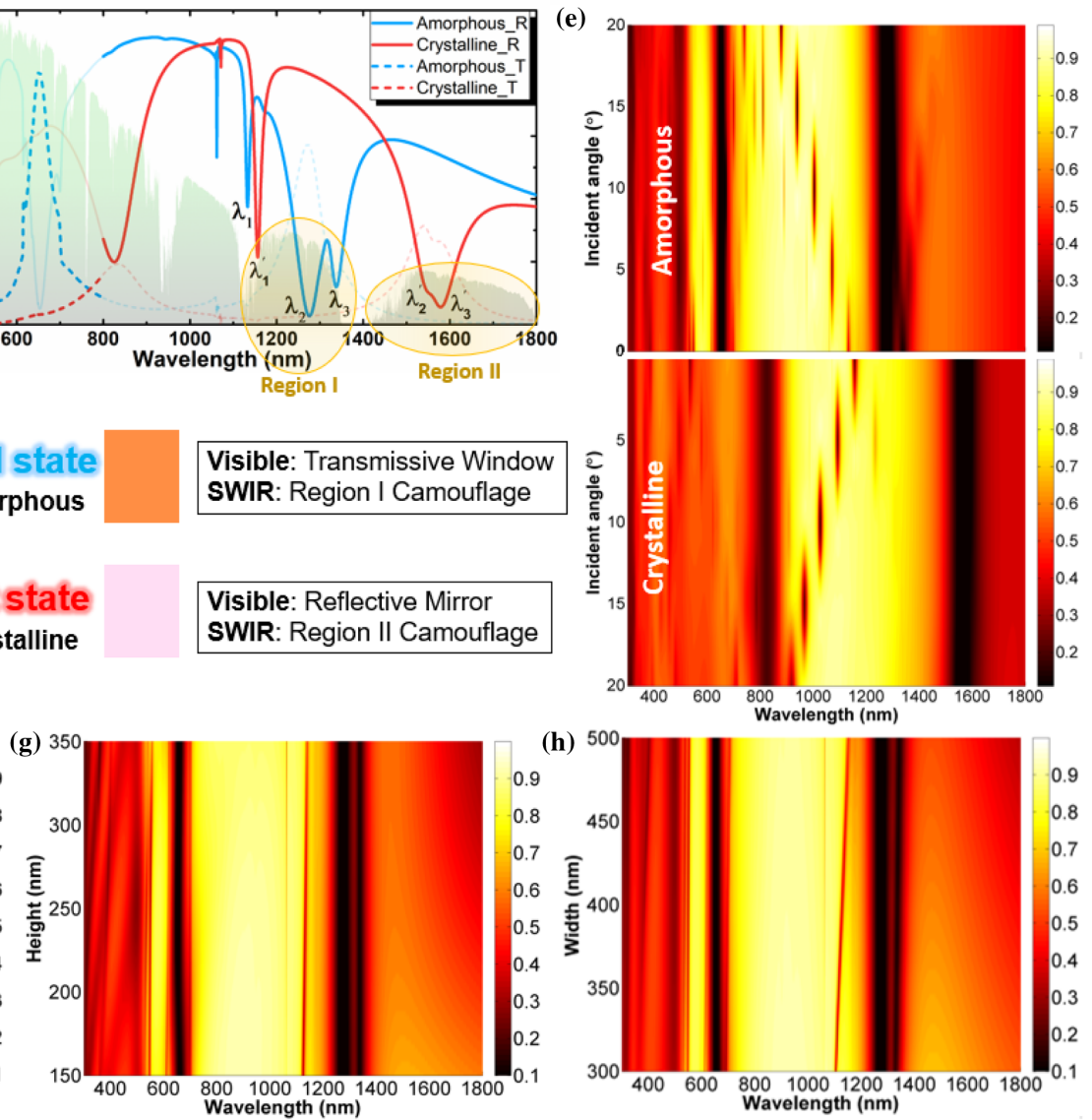

Fig. 2. (a) Schematic representation of the proposed metasurface with its dimensions $\mathrm{P}, \mathrm{W}$, and $\mathrm{H}$. (b) Reflection and transmission data of the metasurface in the amorphous and crystalline phases. The transmission data are bolded in the visible range ( $350-800 \mathrm{~nm})$, and the reflection data are bolded in the SWIR $(900-1800 \mathrm{~nm})$ range. (c) Detection mechanism of the proposed metasurface using a SWIR mode thermal camera. (d) Functionality of the design in the visible and SWIR ranges. The colors shown in this panel are extracted from the Commission Internationale de l'Elcairage (CIE) color map. (e) Angular reflectance response of the metasurface in the amorphous and crystalline phases. The reflection contour plots as a function of the $(\mathrm{f})$ periodicity $(\mathrm{P}),(\mathrm{g})$ grating height $(\mathrm{H})$, and $(\mathrm{h})$ grating width $(\mathrm{W})$. In these plots, the other dimensions are kept in their optimal values.

lateral dimension (W) and its periodicity tunes the spectral position of this resonance mode [see Figs. 2(f)-2(h)]. Looking at the absorption profile of the design, Fig. 3(b) shows that the light is harvested in both the ITO grating and MIM cavity design. The excitation of ESPs in the top metal-insulator interface, with long decay lengths extended throughout the ITO layer (as vectoral profile shows), is responsible for light absorption in the ITO layer. Moreover, the excitation of GMRs within the MIM cavity and ESPs in the bottom metal-insulator interface is responsible for light absorption inside the cavity.

Similar profiles have also been derived for the second resonance mode located at $\lambda_{2}=1275 \mathrm{~nm}$, as shown in Figs. 3(c) and $3(\mathrm{~d})$. Looking at the wavefront of the incoming light, we can see that the design is transparent in the incoming wave. Therefore, this resonance is the transmissive FP mode that is excited in the MIM cavity design. This can also be seen in the extracted contour plots [Figs. 2(f) $-2(h)$ ], where the second resonance spectral position is independent of the grating dimensions.

Finally, this analysis is performed for the third resonance mode at $\lambda_{3}=1338 \mathrm{~nm}$. As shown in Fig. 3(e), in this resonance mode, light is dominantly confined inside the MIM cavity design. The E-field profile in this excitation wavelength resembles the excitation of GMRs inside the cavity. Therefore, in this resonance frequency, the active absorption parts are metal layers of the cavity, as shown in Fig. 3(f).

The amount of absorbed power inside the MIM cavity and ITO layer is calculated using two monitors, which are shown in Fig. 3(g). The absorption spectra within the ITO bulk, the MIM cavity, and the whole design are plotted in Fig. 3(h). As we can see in this panel, in the first resonance mode $(1131 \mathrm{~nm})$, both components are active, which is due to the simultaneous excitation of ESPs and GMRs. In the second resonance mode, MIM is the active part, which is due to the excitation of FP modes inside the cavity. Finally, in the third mode, MIM has the dominant response, while a part of the absorption belongs to the ITO layer. For longer wavelength ranges, i.e., 1400-1700 nm, the only active part is the ITO layer. This non-resonant absorption is due to the bulk absorption within the ITO layer. Finally, the reflected power from the metasurface in the amorphous and crystalline phase is compared with a metal. For this purpose, the reflection spectra of the design are multiplied with the spectral irradiance of a wavelength solar spectrum, as shown in Fig. 4(a). As it can be clearly seen in this figure, the amorphous 

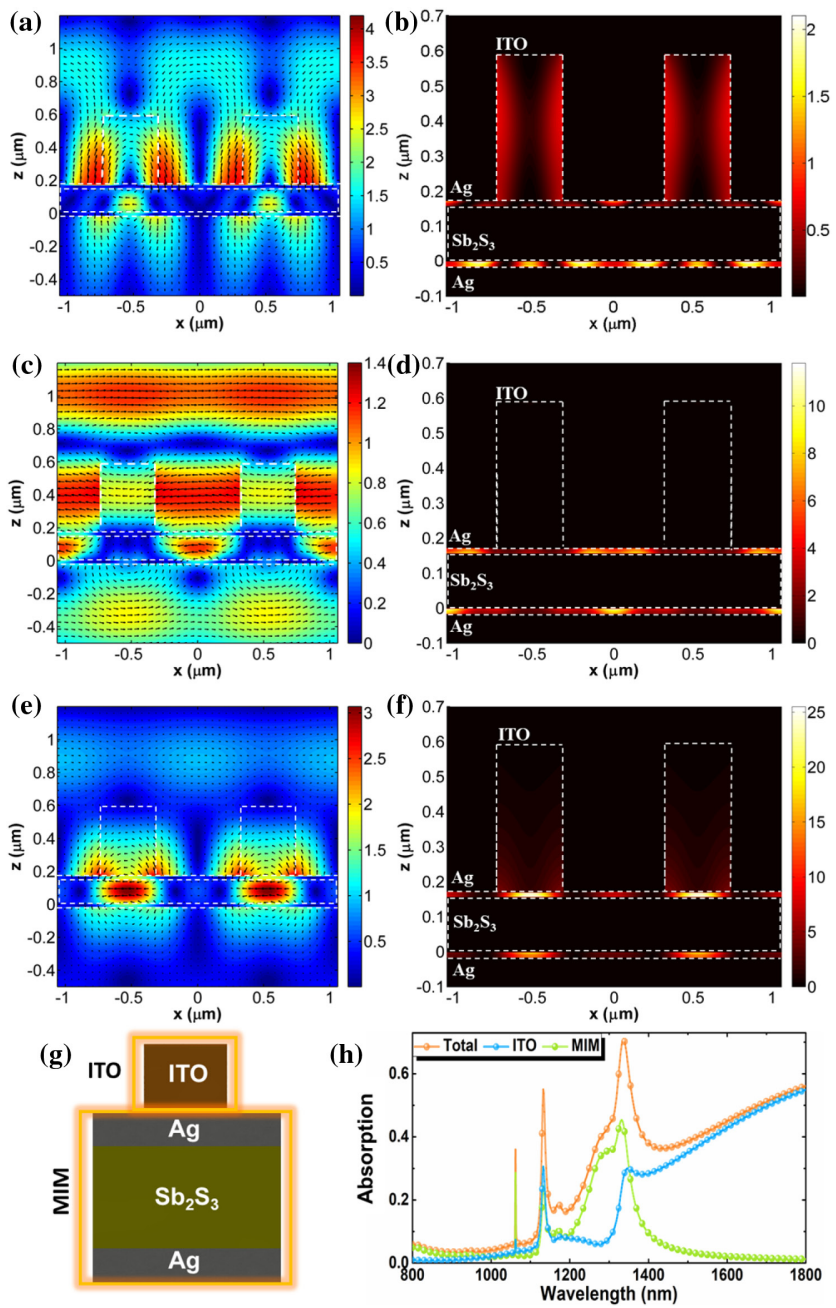

Fig. 3. (a) E-field and (b) absorption profiles across the design in the first resonance mode of $\lambda_{1}=1131 \mathrm{~nm}$ demonstrate the simultaneous excitation of ESPs in the metal-insulator interface and GMRs excited inside the cavity design. (c) E-field and (d) absorption profiles across the design in the first resonance mode of $\lambda_{2}=1275 \mathrm{~nm}$ showing the transmissive FP modes excited in the MIM cavity design. (e) E-field and (f) absorption profiles across the design in the first resonance mode of $\lambda_{3}=1338 \mathrm{~nm}$ indicates the excitation of GMRs inside the cavity. (g) Position of absorption monitors used in our analysis. (h) Absorption profiles within the ITO grating, MIM cavity, and the whole design.

and crystalline phase metasurface has suppressed reflected power in Region I and Region II, respectively. To have a quantitative comparison, the averaged power ratios (compared to an ideal metal reflector) are extracted for both phases in three wavelength ranges of 900-1700, 1150-1350 (Region I), and 1400-1700 nm (Region II), as shown in Fig. 4(b). For this aim, reflected solar power from the design divided to reflect power from the ideal metal reflector is calculated to understand how much reduction is achieved for each phase in the SWIR range from 900 to $1700 \mathrm{~nm}$. The metasurface has reduced reflected power to 0.66 and 0.7 for the amorphous and crystalline phases, respectively. Moreover, the reduction is examined separately to confirm the antireflection capability of phases in different regions. In Region I, this suppression is more pronounced with a ratio of 0.37 for amorphous. For the crystalline phase, the
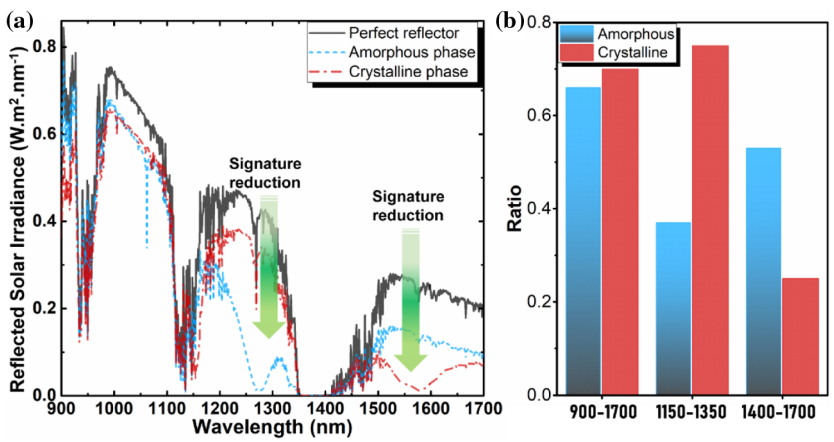

Fig. 4. (a) Reflected solar irradiance from a metal reflector and the metasurface in the amorphous and crystalline phases. (b) Averaged power ratios in three different SWIR ranges.

signature reduction is the most effective in Region II with a ratio of 0.25 .

In summary, in this Letter, an adaptive metasurface design is demonstrated with tunable optical responses in both visible and SWIR ranges. The proposed design acts as a transmissive color filter in the amorphous phase and, upon a temperature increase, it turns into a reflective mirror. In the meantime, this phase transition provides the opportunity for the real-time tuning of the SWIR mode response of the design to cover a broad range and adaptive camouflage.

Disclosures. The authors declare no conflicts of interest.

Data Availability. Data underlying the results presented in this paper are not publicly available at this time but may be obtained from the authors upon reasonable request.

\section{REFERENCES}

1. S. Xiao, T. Wang, T. Liu, C. Zhou, X. Jiang, and J. Zhang, J. Phys. D 53, 503002 (2020).

2. F. Neubrech, X. Duan, and N. Liu, Sci. Adv. 6, eabc2709 (2020).

3. J. He, M. Zhang, S. Shu, Y. Yan, and M. Wang, Opt. Express 28, $37590(2020)$

4. B. Ko, T. Badloe, and J. Rho, ChemNanoMat 7, 713 (2021).

5. Q. He, N. Youngblood, Z. Cheng, X. Miao, and H. Bhaskaran, Opt. Express 28, 39841 (2020).

6. M. Jafari, L. J. Guo, and M. Rais-Zadeh, Adv. Opt. Mater. 7, 1801214 (2019).

7. H. Liu, W. Dong, H. Wang, L. Lu, Q. Ruan, Y. S. Tan, R. E. Simpson, and J. K. W. Yang, Sci. Adv. 6, 1 (2020).

8. M. Delaney, I. Zeimpekis, D. Lawson, D. W. Hewak, and O. L. Muskens, Adv. Funct. Mater. 30, 2002447 (2020).

9. R. Hu, S. Huang, M. Wang, X. Luo, J. Shiomi, and C. W. Qiu, Adv. Mater. 31, 1807849 (2019).

10. E. Buhara, A. Ghobadi, B. Khalichi, H. Kocer, and E. Ozbay, J. Phys. D 54, 265105 (2021).

11. L. Xiao, H. Ma, J. Liu, W. Zhao, Y. Jia, Q. Zhao, K. Liu, Y. Wu, Y. Wei, S. Fan, and K. Jiang, Nano Lett. 15, 8365 (2015).

12. A. Kalantari Osgouei, H. Hajian, B. Khalichi, A. E. Serebryannikov, A. Ghobadi, and E. Ozbay, Plasmonics 16, 1013 (2021).

13. J. Song, S. Huang, Y. Ma, Q. Cheng, R. Hu, and X. Luo, Opt. Express 28, $875(2020)$

14. W. Xi, Y. Liu, J. Song, R. Hu, and X. Luo, Opt. Lett. 46, 888 (2021).

15. A. Kalantari Osgouei, A. Ghobadi, B. Khalichi, and E. Ozbay, J. Opt. 23, 085001 (2021).

16. P. B. Johnson and R. W. Christy, Phys. Rev. B 6, 4370 (1972).

17. Lumerical Solution Inc., http://www.Lumerical.Com/TcadProducts/Fdtd/.

18. I. Abdulhalim, Nanophotonics 7, 1891 (2018).

19. B. Khalichi, A. Ghobadi, A. Kalantari Osgouei, and E. Ozbay, J. Phys. D 54, 365102 (2021). 